Research Article

\title{
Crossing Reliability of Electric Bike Riders at Urban Intersections
}

\author{
Huan Mei, Yang Xiaobao, and Jia Bin \\ MOE Key Laboratory for Urban Transportation Complex Systems Theory and Technology, Beijing Jiaotong University, \\ Beijing 100044, China \\ Correspondence should be addressed to Yang Xiaobao; yangxb@bjtu.edu.cn
}

Received 13 June 2013; Accepted 31 July 2013

Academic Editor: Wuhong Wang

Copyright (C) 2013 Huan Mei et al. This is an open access article distributed under the Creative Commons Attribution License, which permits unrestricted use, distribution, and reproduction in any medium, provided the original work is properly cited.

\begin{abstract}
This paper presents a crossing reliability model of electric bike riders at urban intersections using survival analysis approach. Riders' crossing behavior was collected by video cameras. Waiting times in the red-light phase were modeled by reliability-based model that recognizes the covariate effects. Three parametric models by the exponential, Weibull, and log-logistic distributions were proposed to analyze when and why electric bike riders cross against the red light. The results indicate that movement information and situation factors have significant effects on riders' crossing reliability. The findings of this paper provide an important demonstration of method and an empirical basis to assess crossing reliability of electric bike riders at the intersection.
\end{abstract}

\section{Introduction}

Nonmotorized vehicles (i.e., mainly regular bicycles and electric bikes) are one of the most popular means of transportation in some Asian developing countries, such as Vietnam, Cambodia, and China. Even in developed countries, cycling travel is recognized as low energy consumption, healthy to the users and do not damage the health of others. For example, people who go to work by bike would obtain material reward from the government in Korea [1]. In London, in order to encourage people to travel by bike, the local government carried out the cycling revolution [2].

In recent years, the electric bike has entered people's life. Because of electric bike is labor-saving and speedy, it has emerged as a popular mode of transportation in many large cities in China [3]. The number of Chinese electric bikes was about 140 million in 2012 [4]. Electric bikes in China are defined as electric two wheelers with relatively low speeds and weights compared to a motorcycle. Both bicyclestyle electric bikes (with functioning pedals) and scooterstyle electric bikes (with many of the features of gasoline scooters) are classified as bicycles and are given access to bicycle infrastructure (see Figure 1).

However, the growing popularity of cycling traffic also entails safety concerns as observed in accident and injury statistics. Traffic accident proportion of cyclists has always been high because cyclists are vulnerable groups in the traffic conflict. In 2010, 4616 regular bicyclists were killed and 14,283 were seriously injured in road accidents, representing $7.1 \%$ of all traffic fatalities and $5.6 \%$ of injuries [5]. With the rapidly increasing number of electric bikes, more and more people pay much concerned about traffic security problems involved electric bikes. In 2004, 589 electric bike riders were died and 5295 were seriously injured in road accidents [6]. In 2010, the corresponding figures increased to 4029 and 20,311, respectively, representing $6.2 \%$ of all traffic fatalities and $8.0 \%$ of injuries [5].

Accident analysis reveals that over $60 \%$ of cyclist fatal crashes were caused by violation of traffic rules [5]. Redlight crossing is a typical type of rule violation behavior. Because of the poor law enforcement and peoples' low safety awareness, violation behavior in the red-light period is rather prevalent and represents a substantial safety problem in Chinese urban intersections [7]. Particularly, electric bike riders with relatively high speed are much more likely to increase the risk of traffic incident.

So far, many scholars have studied red-light crossing behaviors, but many focused on motorized vehicles $[8,9]$ and pedestrians [10-13]. Unfortunately, only a few studies have investigated bicyclists' red-light crossing behavior, much less 

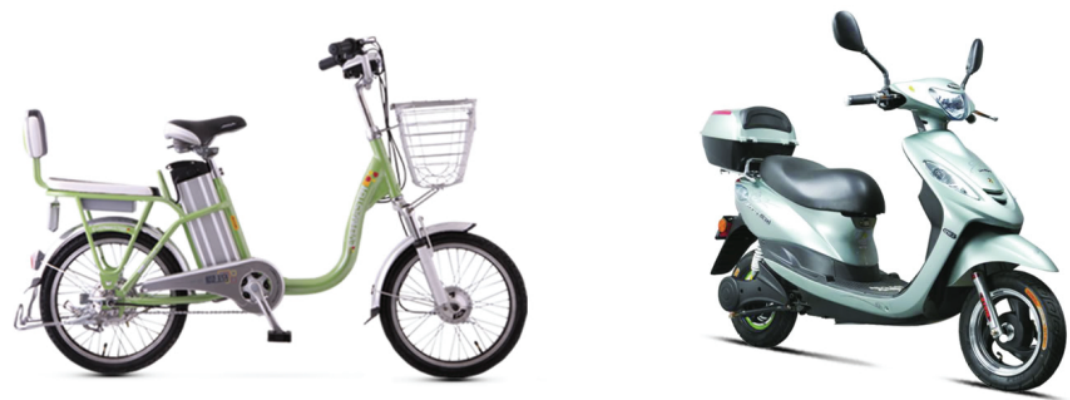

Figure 1: Bicycle-style electric bike at the left and scooter-style electric bike at the right.

about electric bike riders. Johnson et al. analyzed the bicycle violation behavior at intersections in Melbourne [14]. They found out that the rate of red-light crossing before noon was $3 \%$ and $11 \%$ in the afternoon. Johnson et al. collected 4225 bicyclists who arrived at the intersections at red-light time, and their result indicated that $6.9 \%$ bicyclists violated traffic rules [15]. Wu et al. used logistic model to analyze how gender, age, and conformity behavior affected the cyclist's violation, and classify red-light crossing behavior to three types: obey the rules, risk taking, opportunistic [7]. Johnson et al. obtained the data through the Internet and used TPB (theory of planned behavior) to investigate the bicyclists' red-light crossing behavior and attitude and the influencing factors in Australia [16]. Their result showed that $37.3 \%$ of the riders violated traffic rules, and the violation rate of males was higher than females, the young was higher than the old, and a rider who was accident-free may violate them easier.

Most of the existing researches on cyclists' crossing behavior used logistic model to analyze the violating probability and its influence factors. And little effort was focused on the red-light crossing behavior of electric bike riders. In this paper, we use survival analysis method to study electric bike riders' crossing reliability at the signalized intersection. Survival analysis is a common topic in many areas including biomedical, engineering, and social sciences. In the transportation field, it has been applied to describe a number of time-related issues including activity duration $[17,18]$, traffic accidents [19], and vehicle travel time [20, 21]. Hazard-based duration models of survival analysis have an advantage in that it allows the explicit study of the relationship between duration time and the explanatory variables [22-24]. More importantly, survival analysis models can deal with not only uncensored data but also censored data. For example, the exact waiting duration reflecting cyclist endurance cannot be observed if cyclists wait until the permission of traffic rules. This is the very reason why survival analysis method is chosen to analyze riders' crossing behavior. The empirical data, which were obtained by video cameras, are modeled by three typical parametric hazard functions. Both crossing reliability and waiting times of electric bike riders under various conditions are calculated and the covariate effects are quantified. The finding of this paper can explain when and why electric bike riders violate traffic light at the intersection. It is hoped that the results can help to improve the planning and designing of signalized intersections in developing countries.

\section{Method}

Reliability is the probability that a system or component will perform its required function under stated conditions for a specified period of time. Accordingly, crossing reliability of electric bike riders can be defined as the probability that an electric bike rider obey the traffic light after a specified waiting time at the signalized intersection.

Let $T$ denote a nonnegative random variable representing the failure time or time-to-failure. Mathematically, the reliability function $R(t)$ is the probability that a system will be successfully operating without failure in the interval from time 0 to time $t$,

$$
R(t)=P(T>t), \quad t>0 .
$$

In this paper, it is assumed that an electric bike rider violate the traffic light when his/her waiting duration time is $T$. $R(t)$ is the crossing reliability of electric bike riders. It is the probability that a rider, who arrives at the intersection in the red-light period, will not violate the traffic light in his/her waiting duration time from 0 to $t$.

The failure probability, or unreliability of riders' crossing behavior, is then

$$
F(t)=1-R(t)=P(T \leq t),
$$

which is known as the distribution of $T$.

If the random variable $T$ has a density function $f(t)$, then

$$
R(t)=\int_{t}^{\infty} f(x) d x .
$$

The density function can be mathematically described as $\lim _{\Delta t \rightarrow 0} P(t \leq T<t+\Delta t)$. This can be interpreted as the probability that the failure/violation time will occur between time $t$ and the next interval of waiting time, $t+\Delta t$. The three functions, $R(t), F(t)$, and $f(t)$ are closely related to one another. If any of them is known, all the others can be determined.

The waiting time of an electric bike rider in the red-light period can be regarded as the waiting duration that starts when a cyclist arrives at the intersection in the red period and ends when the rider starts to cross the intersection. Therefore, hazard-based duration model in survival analysis can be adopted to calculate the probability of rider violation 
with different waiting durations. In survival analysis, $R(t)$ is also called survival function or survivor probability. The survival function is defined to be the probability that the waiting time of a rider in a red-light is longer than a specific time, $t$.

Another important function in survival analysis is the hazard function, $h(t)$. The hazard function in this paper is the instantaneous rate at which the waiting duration will end in an infinitesimally small time period, $\Delta t$, after time $t$, given that the duration time has lasted to time $t$

$$
\begin{aligned}
h(t) & =\lim _{\Delta t \rightarrow 0} \frac{P(t<T \leq t+\Delta t \mid T>t)}{\Delta t} \\
& =\lim _{\Delta t \rightarrow 0} \frac{P(t<T \leq t+\Delta t)}{\Delta t \times P(T>t)}=\frac{f(t)}{R(t)} .
\end{aligned}
$$

The importance of the hazard function is that it indicates the changing rate in the red-light violating behavior over the waiting period of a rider. For example, two riders may have the same crossing reliability at a specific point in time, but the hazard rate curves can be very different.

Note that crossing reliability of electric bike riders is influenced by various factors. The influential factors can be defined as a vector of explanatory variables, $\mathbf{x}=$ $\left(x_{1}, x_{2}, \ldots, x_{m}\right)^{\prime}$. To accommodate the effects of these influential factors, a parametric hazard model or a semiparametric hazard model may be adopted. If little or no knowledge of the functional form of the hazard is available, a semiparametric approach for modeling the hazard function is convenient. If theoretical support for a parametric shape is available, using a particular parametric distributional form for the hazard function will be appropriate. Both semiparametric and fully parametric hazard-based models have been widely cited in the literature. For detailed discussion of different approaches for duration models see Bhat [17], Lee and Wang [25]. Here, the primary objective of this paper is to examine the crossing reliability and consider the application of the model. A parametric hazard approach is adopted because its hazard function can be chosen flexibly. Several typically parametric distributions are exponential, Weibull, and loglogistic. These common distributions are summarized below.

The exponential distribution is suitable for modeling data with constant hazard. The exponential hazard and survivor functions are

$$
\begin{gathered}
h(t)=\lambda(\text { constant hazard }), \\
R(t)=\exp (-\lambda t) .
\end{gathered}
$$

The model is implemented by parameterizing $\lambda_{j}=\exp \left(-X_{j}\right.$ $\beta$ ) when the influenced covariates are considered. Let $h_{i}\left(t, \lambda_{i}\right)$ and $h_{j}\left(t, \lambda_{j}\right)$ be the hazards of individuals $i$ and $j$; the hazard ratio of these two individuals is

$$
\frac{h_{i}\left(t, \lambda_{i}\right)}{h_{j}\left(t, \lambda_{j}\right)}=\frac{\lambda_{i}}{\lambda_{j}}=\exp \left[-\sum_{k} \beta_{k}\left(x_{k i}-x_{k j}\right)\right] .
$$

This ratio is dependent only on the differences of the covariates of the two individuals and the coefficients. It does not depend on the time $t$.
The Weibull distribution is suitable for modeling data with monotone hazard rates that either increase or decrease exponentially with time. The Weibull hazard and survivor functions are

$$
\begin{gathered}
h(t)=\lambda \gamma(\lambda t)^{\gamma-1}, \\
R(t)=\exp \left(-(\lambda t)^{\gamma}\right) .
\end{gathered}
$$

The model is implemented by parameterizing $\lambda_{j}=\exp \left(-X_{j}\right.$ $\beta$ ), where $\gamma$ is an ancillary parameter to be estimated from the data. Note that the hazard increases with duration time if $\gamma>1$, decreases if $\gamma<1$, and reduces to exponential if $\gamma=1$. Similar to the exponential distribution model, the hazard ratio of the Weibull distribution model is also not time dependent.

The log-logistic model assumed that the natural logarithm of time follows a logistic distribution. The log-logistic hazard and survivor functions are

$$
\begin{gathered}
h(t)=\frac{\lambda \gamma(\lambda t)^{\gamma-1}}{\left\{1+(\lambda t)^{\gamma}\right\}}, \\
R(t)=\left\{1+(\lambda t)^{\gamma}\right\}^{-1} .
\end{gathered}
$$

The model is implemented by parameterizing $\lambda_{j}=\exp \left(-X_{j}\right.$ $\beta$ ) and treating the scale parameter $\gamma$ as an ancillary parameter to be estimated from the data. Unlike the exponential and Weibull distributions, the log-logistic distribution is indicated for data exhibiting non-monotonic hazard rates. When $\gamma>1$, the hazard initially increases and then decreases with duration time. When $0<\gamma \leq 1$, the hazard decreases with duration time. The hazard ratio of the log-logistic distribution model is time dependent.

In order to keep the linear form of the covariates, three new terms can be defined:

$$
\begin{gathered}
y_{i}=\log t_{i}=\beta X+\varepsilon_{i} \sigma, \\
\varepsilon=\frac{(\log t-\beta X)}{\sigma}, \\
\gamma=\frac{1}{\sigma} .
\end{gathered}
$$

The likelihood and log-likelihood functions for $N$ observations on $y_{i}$ and right censoring indicator $\delta_{i}$ are

$$
\begin{gathered}
L=\prod_{i}\left[\sigma^{-1} f\left(\varepsilon_{i}\right)\right]^{\delta_{i}}\left[R\left(\varepsilon_{i}\right)\right]^{1-\delta_{i}} \\
\log L=\sum_{i}\left[\delta_{i}\left(-\log \sigma+\log f\left(\varepsilon_{i}\right)\right)+\left(1-\delta_{i}\right) \log R\left(\varepsilon_{i}\right)\right] .
\end{gathered}
$$

Log-likelihood functions can be maximized using any standard method (e.g., Newton; BFGS).

\section{Data}

The field observation approach was used in this study. It has been widely used to study pedestrian and cyclist behaviors 
TABLE 1: Covariates selection and explanation.

\begin{tabular}{|c|c|c|}
\hline Covariate & Type & Explanation \\
\hline AGE (age group) & Continuous variable & $\begin{array}{l}1 \text { if under } 20,2 \text { if } 20-29,3 \text { if } 30-39,4 \text { if } 40-49,5 \text { if } \\
50-59 \text {, and } 6 \text { otherwise }\end{array}$ \\
\hline GEN (gender) & Binary indicator & 1 if male, 0 female \\
\hline WN (waiting number) & Continuous variable & $\begin{array}{l}\text { The number of other cyclists that are waiting for a green } \\
\text { light when arrives }\end{array}$ \\
\hline $\mathrm{CN}$ (crossing number) & Continuous variable & $\begin{array}{l}\text { The number of other cyclists that are crossing against } \\
\text { the red light when arrives }\end{array}$ \\
\hline TC (twice crossing) & Binary indicator & 1 if twice crossing behavior, 0 otherwise \\
\hline MV (motor vehicle volume) & Continuous variable & $\begin{array}{l}\text { Motor vehicle volume per lane per min in red-light } \\
\text { phase when the rider arrives }\end{array}$ \\
\hline LT (left turn phase) & Binary indicator & $\begin{array}{l}1 \text { if an electric bike rider violates traffic rules in the } \\
\text { left-turn period of motorized vehicles, } 0 \text { otherwise }\end{array}$ \\
\hline YL (yellow light) & Binary indicator & $\begin{array}{l}1 \text { if an electric bike rider violates traffic rules in the } \\
\text { yellow light period, } 0 \text { otherwise }\end{array}$ \\
\hline
\end{tabular}

in actual traffic situations [7, 13, 26, 27]. For example, Tiwari et al. used video recording to analyze pedestrians' violating behavior in signalized intersections in India [13]. Yao et al. also used video cameras to study the behavior characteristics of pedestrian crowd weaving flow in transport terminal [27]. Wu et al. used video recordings to examine urban commuter cyclists' red-light running behaviors in Beijing [7]. Wang et al. used video data to study pedestrians' crossing behavior in red-light period in Beijing [26].

3.1. Site Characteristics. A cross-sectional observational study was conducted at five signalized intersections in Beijing. Three criteria were used to select the observational sites. First, the selected sites should represent the typical intersection design characteristics and traffic conditions of urban areas in Beijing. Second, the selected intersections should have similar characteristics involved geometrics, traffic conditions, traffic control, and the absence of pointsmen. In addition, there have to be a reasonably high number of electric bike traffic during the observation period.

3.2. Data Collection and Processing. Video cameras were used to collect data of electric bike riders' crossing behaviors at signalized intersections. The cameras were carefully placed so that the road users were unaware that they were being observed. The data collection was conducted on weekdays during daylight hours (i.e., 8:00 a.m. to 5:30 p.m.) in good weather conditions.

All road users who entered the intersection were recorded on video, but only the riders arriving in red-light phases were coded. In addition, only the straight-going riders crossing through the intersection were recorded. Left-turners and right-turners of electric bike were excluded because of the limited field of view of the cameras. The waiting duration was from the time a rider arrived at the stop line to the time he/she began to cross. It can be classified into two kinds: uncensored data and censored data. Uncensored data is defined as the waiting duration which ends within the red-light period (violating crossing). Otherwise, the waiting duration is called censored data as long as it ends within the green light period (normal crossing). For censored data, it is unknown about his/her exact maximum endurance of waiting time.

Considering the previous researches and intuitive arguments regarding the influential factors of cyclist crossing behavior, three sets of variables were coded (see Table 1). The first set described the riders' individual characteristics, including gender and age. The second set of variables focused on the riders' movement information, including twice crossing behavior, the times of arrival at and departure from the stop line, the time when crossing is completed, the status of the traffic light at each of three times, and whether the violating behavior occurs in the left-turn period of motorized vehicles or the yellow-light period. The last set of variables of concern were situation factors, including the red-light cycle length, the number of riders waiting upon arrival, the number of riders crossing against the red-light, and crossing traffic volume (i.e., the number of motorized vehicles that crossed the intersection from either direction during the time of the red-light cycle when the rider arrives).

\section{Results}

A total of $8 \mathrm{~h}$ of video recordings (approximately $1.5 \mathrm{~h}$ for each site) were collected and 1946 crossing events of cyclists were observed. Demographics and behavioral data were coded only for electric bike riders during the red-light phases. Thus, a total of $312(16 \%)$ valid observations were obtained (see Table 2).

4.1. Descriptive Statistics. The proportions of red-light crossing of electric bike riders in each sub-group were presented in Table 2. Of all the electric bike riders we observed, the number of males was larger than females ( $84.3 \%$ versus $15.7 \%)$. And most of the electric bike riders are young and middle-aged persons; the respective rates are $26.5 \%$ and $67.6 \%$. The old riders only occupied $6.1 \%$.

The overall proportion of electric bike riders who crossed against the red-light was $75.4 \%$. Male electric bike riders 
TABLE 2: Red-light crossing rates and waiting times by each subcategory.

\begin{tabular}{|c|c|c|c|c|c|c|}
\hline & \multicolumn{2}{|c|}{ Violating crossing } & \multicolumn{2}{|c|}{ Normal crossing } & \multicolumn{2}{|c|}{ Overall } \\
\hline & Rates & Waiting time (s) & Rates & Waiting time (s) & Rates & Waiting time (s) \\
\hline \multicolumn{7}{|l|}{ Gender } \\
\hline Male & $76.9 \%$ & 26.11 & $23.1 \%$ & 64.88 & $84.3 \%$ & 35.06 \\
\hline Female & $67.3 \%$ & 22.11 & $32.7 \%$ & 69.14 & $15.7 \%$ & 37.46 \\
\hline \multicolumn{7}{|l|}{ Age group } \\
\hline Young $(<30)$ & $80.7 \%$ & 24.83 & $19.3 \%$ & 82.57 & $26.5 \%$ & 35.96 \\
\hline Middle (30-50) & $74.4 \%$ & 26.67 & $25.6 \%$ & 59.46 & $67.6 \%$ & 35.12 \\
\hline Old $(>50)$ & $63.2 \%$ & 13.78 & $36.8 \%$ & 75.97 & $6.1 \%$ & 36.69 \\
\hline Overall & $75.4 \%$ & 25.55 & $24.6 \%$ & 65.76 & $100 \%$ & 35.44 \\
\hline
\end{tabular}

TABLE 3: Parameter regression values of the influence variables.

\begin{tabular}{lcccccccc}
\hline Parameter & \multicolumn{3}{c}{ Exponential model } & \multicolumn{3}{c}{ Weibull model } & \multicolumn{3}{c}{ Log-logistics model } \\
& Coef. & Haz. ratio & $P$ value & Coef. & Haz. ratio & $P$ value & Coef. & $P$ value \\
\hline Constant & 2.960 & 0.052 & $<0.001$ & 1.009 & 0.364 & 0.084 & 1.449 & 0.359 \\
SITE & 0.145 & 0.865 & 0.077 & 0.044 & 0.957 & 0.543 & 0.012 & 0.951 \\
AGE & -0.065 & 1.067 & 0.424 & -0.055 & 1.057 & 0.485 & -0.220 & 0.329 \\
GEN & 0.012 & 0.988 & 0.954 & 0.040 & 0.961 & 0.841 & 0.197 & 0.737 \\
WN & 0.041 & 0.960 & 0.018 & 0.064 & 0.938 & $<0.001$ & 0.266 & $<0.001$ \\
CN & -0.134 & 1.143 & $<0.001$ & -0.089 & 1.094 & $<0.001$ & -0.382 & $<0.001$ \\
TC & -0.206 & 1.229 & 0.254 & -0.331 & 1.392 & 0.049 & -1.151 & 0.017 \\
MV & 0.189 & 0.828 & 0.001 & 0.146 & 0.864 & 0.006 & 0.429 & 0.006 \\
LT & -1.166 & 3.209 & $<0.001$ & -0.95 & 2.585 & $<0.001$ & -2.431 & $<0.001$ \\
YL & -0.737 & 2.090 & 0.002 & -0.882 & 2.415 & $<0.001$ & -3.065 & $<0.001$ \\
$\gamma$ & 1.000 & & & 0.408 & & & 0.514 & -671.2 \\
\hline $\log L$ & & -831.6 & & & -656.6 & & & \\
\hline
\end{tabular}

were found to be more likely to violate traffic rules at signal intersections than females (76.9\% versus 67.3\%). Red-light violation rates also differed by age group. Young- and middleaged riders were more likely to run against a red-light than the old ones $(80.7 \%$ and $74.4 \%$ versus $63.2 \%)$. The result is consistent with the research in pedestrian crossing behavior $[13,23,26]$.

The average waiting time of all samples was 35.44 seconds. The average waiting time of the violating crossing was 25.55 seconds, while the average waiting time of the normal crossing is 65.76 seconds. The maximum waiting duration was 161 seconds while the minimum was 0 second. The latter means people cross the street without any waiting time. As shown in the last column of Table 2 , the overall waiting times of electric bike riders vary from 35.06 to 37.46 seconds in different genders, and from 35.12 to 36.99 seconds in different age groups. Therefore, there is no significant difference of the overall waiting times in gender and age group.

This descriptive statistic cannot reflect the exact waiting behavior due to the neglect of the censored data. The estimation of the waiting duration with censored data will be discussed later.
4.2. Parameter Regression Analysis. Table 3 shows the estimated parameters of the parametric hazard models in predicting red-light crossing behavior of electric bike riders. Three models show perfect overall goodness-of-fit indicated by the log-likelihood values. Among them, the Weibull distribution is considered the best to describe riders' crossing behavior. The hazard ratios of both the exponential and Weibull models are not time-dependent. According to (6), the hazard ratio is $\exp \left(-\beta_{i}\right)$ times if the $i$ th covariate increases by one unit. However, the risk of the log-logistic model cannot be calculated by this way, since it is time dependent.

From the estimated results, the site variable is insignificant, which means the selected intersections have similar traffic and geometric characteristics. Besides, gender and age group have low significant level. It is partly because of the low proportions of female $(15.7 \%)$ and old people $(6.1 \%)$ in the sample. Most of other covariates are statistically significant at the 0.10 level of significance. These covariates are significantly related to violation behavior.

For the specific parameter estimation in Table 2, based on (9), the positive parameters have a positive effect on waiting time of riders (e.g., WN and MV). Therefore, an increase 


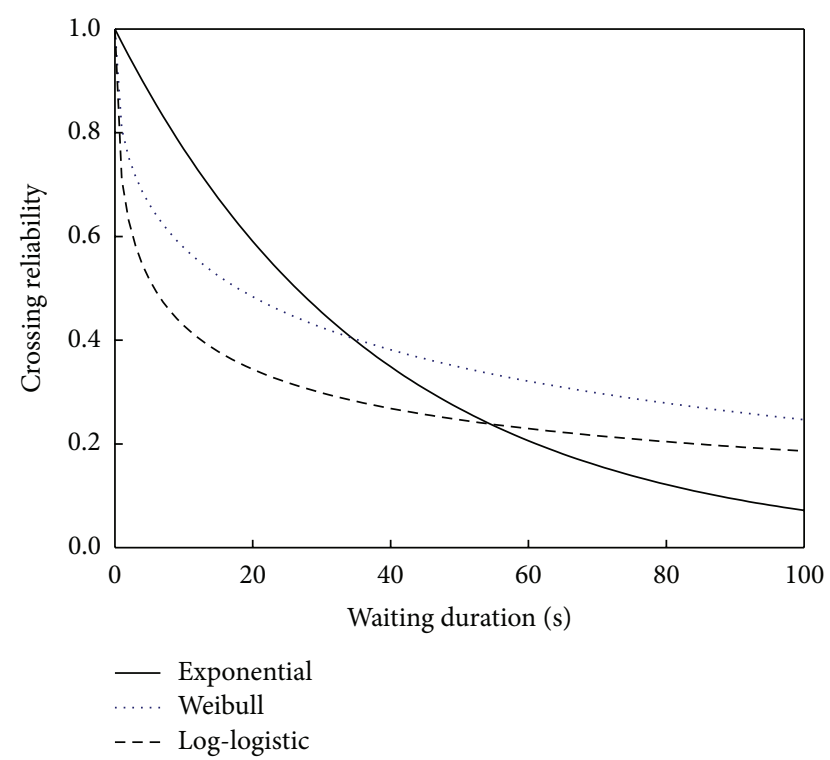

FIgURE 2: Crossing reliability with waiting duration in different parametric models.

in the corresponding covariates can increase the waiting duration or decrease the risk rate. For example, an electric bike rider is willing to wait longer time if many other cyclists are waiting when he/she arrives at the intersection. Also, a rider is likely to wait to reduce the collision risk in the presence of heavy traffic.

On the contrary, the negative effect means that an increase in the corresponding covariates can decease the waiting duration or increase the risk rate (e.g., CN, TC, LT, and YL). For example, an electric bike rider may follow to violate rules when other cyclists are crossing against the red-light. A rider of twice crossing has higher hazard and shorter waiting time. Besides, a rider is more likely to violate rules during the leftturn period of motorized vehicles and the yellow-light period.

4.3. Crossing Reliability Analysis of Electric Bike Riders. Crossing reliability of electric bike riders calculated by three parametric distributions are shown in Figure 2. All of the probability curves present a monotonically decreasing trend, which means the crossing reliability decreases with the increasing waiting time. In addition, the curves of the Weibull and log-logistic distributions have a rapidly decreasing trend in the early part of the waiting time. It is consistent with the fact that about $28.1 \%$ of electric bike riders are at high risk of violation and low waiting time to cross against the red right in our sample. But, the exponential distribution has a low adaptability to describe the waiting time of electric bike riders.

Figure 3 gives the risk rate curve calculated by three parametric models. The constant risk in the exponential model reflects the fact that the risk of violating behavior would not change with the elapse of waiting time. Therefore, the exponential model is not appropriate for describing the crossing behavior of electric bike riders. On the other hand,

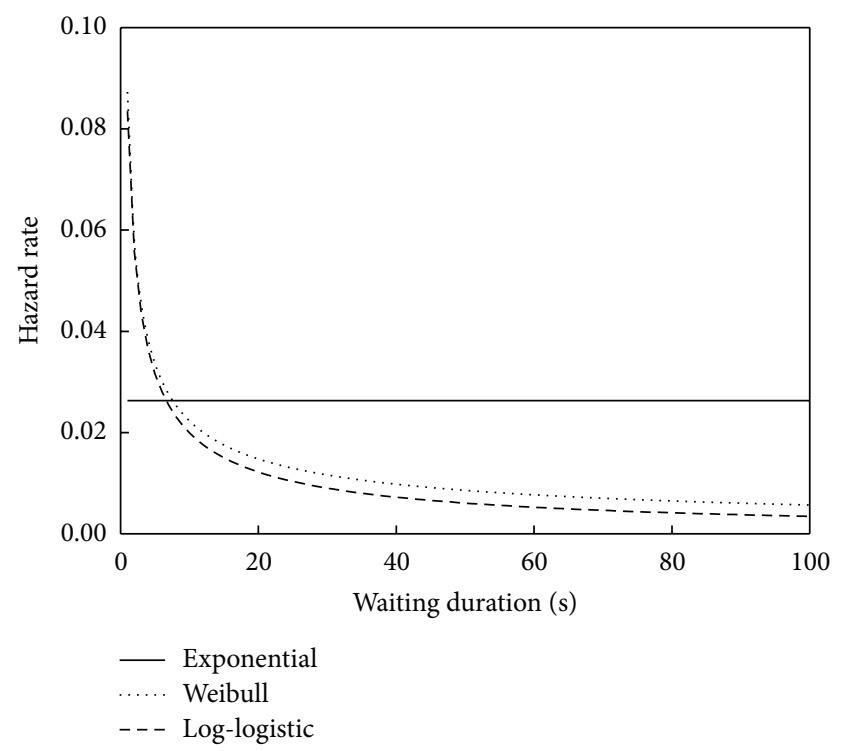

FIGURE 3: Hazard rate with waiting duration in different parametric models.

the curves of the Weibull and log-logistic distributions show a clear downward trend over waiting time, reflecting a negative dependence. It means that the likelihood of terminating the waiting time decreases with elapsed waiting time. It is consistent with the fact that a part of riders are generally nonrisk takers who can obey the traffic rules after waiting a long time.

4.4. Reliability Function of Crossing Behavior. Once electric bike riders violate traffic light, such a crossing behavior is defined as the occurrence of a risk event. Crossing reliability of electric bike riders is the probability that an electric bike rider obey the traffic light after a specified waiting time at the intersection. The Weibull distribution model gives the best description of riders' crossing behavior. Taken the Weibull distribution model, crossing reliability of electric bike riders using our sample data can be written as

$$
\begin{aligned}
R(t)=\exp \left(-(\lambda t)^{\gamma}\right)= & \exp \left\{-[t \exp (-\beta X)]^{\gamma}\right\} \\
=\exp \{-[t \exp ( & 1.009-0.064 * W N+0.089 * C N \\
& +0.331 * T C-0.146 * M V \\
& \left.+0.95 * L T+0.880 * Y L)]^{0.408}\right\} .
\end{aligned}
$$

The proposed reliability model can capture the covariate effects of crossing behavior of electric bike riders at the intersection. Before the applications, however, it is noted that the model should be estimated using the specified field data. Additionally, the explanatory variables should be chosen flexibly according to the specified traffic circumstance. 


\section{Conclusions}

The paper uses the concept of crossing reliability and the methodology of survival analysis to examine violating behavior of electric bike riders at the intersection. Three parametric duration models by the exponential, Weibull, and log-logistic distributions are proposed to analyze the covariate effects on crossing reliability of electric bike riders in red-light phase. The methodology uses a duration model structure that is able to predict crossing reliability at any given traffic conditions. More importantly, three sets of variables are selected as the covariates: individual characteristics, movement information, and situation factors. It is proved that the Weibull distribution is the most appropriate for describing crossing behavior of electric bike riders.

The paper provides several important insights into the determinants of crossing reliability of electric bike riders. Firstly, from the methodological standpoint, this study has provided the empirical evidence that hazard-based duration approach is appropriate for crossing reliability analysis. A statistical test of covariate effects on crossing reliability clearly indicates the goodness-of-fit of the used parametric hazard models. Secondly, the results indicate that movement information and situation factors are significantly related to riders' crossing reliability. Finally, various factors can modify crossing reliability of electric bike riders. The more riders waiting at the stop line, the less other riders crossing against the red-light, and the more motorized vehicle volume, electric bike riders would endure longer waiting times and have higher crossing reliability. While, electric bike riders have lower crossing reliability during the left-turn period of motorized vehicles and the yellow-light period.

In terms of the future work, research with more datasets is required. Also, more parameters under different situations should be taken into account. In addition, it is necessary to study the comparison between electric bikes and common bikes. It is expected that the findings will help traffic engineers, urban planners, and policy makers to understand electric bike riders' behavior at signalized intersections.

\section{Acknowledgments}

This work was supported by the National Basic Research Program of China (Grant no. 2012CB725401), the National Natural Science Foundation of China (Grant nos. 70901005, 71131001), and Fundamental Research Funds for the Central Universities (Grant no. 2013JBM043).

\section{References}

[1] http://zhaokuang541020.blog.163.com/blog/static/127930106201021931336293/.

[2] http://www.nea.gov.cn/2012-06/20/c_131664583.htm.

[3] Q. Zhou, H. Lu, and W. Xu, "Comparison and analysis of urban resident trip characteristics," Journal of Central South Highway Engineering, vol. 32, no. 2, pp. 145-149, 2007 (Chinese).

[4] http://www.cebike.com/news/html/201204/2012042908414362 .htm.
[5] CRTASR, China Road Traffic Accidents Statistics Report, Traffic Administration Bureau of China State Security Ministry, Beijing, China, 2010, (Chinese).

[6] CRTASR, China Road Traffic Accidents Statistics Report, Traffic Administration Bureau of China State Security Ministry, Beijing, China, 2004, (Chinese).

[7] C. X. Wu, L. Yao, and K. Zhang, "The red-light running behavior of electric bike riders and cyclists at urban intersections in China: an observational study," Accident Analysis and Prevention, vol. 49, no. 11, pp. 186-192, 2012.

[8] K. L. H. Martinez and B. E. Porter, "Characterizing red light runners following implementation of a photo enforcement program," Accident Analysis and Prevention, vol. 38, no. 5, pp. 862-870, 2006.

[9] B. E. Porter, K. L. Johnson, and J. F. Bland, "Turning off the cameras: red light running characteristics and rates after photo enforcement legislation expired," Accident Analysis and Prevention, vol. 50, pp. 1104-1111, 2013.

[10] M. M. Ishaque and R. B. Noland, "Behavioural issues in pedestrian speed choice and street crossing behaviour: a review," Transport Reviews, vol. 28, no. 1, pp. 61-85, 2008.

[11] E. Papadimitriou, G. Yannis, and J. Golias, "A critical assessment of pedestrian behaviour models," Transportation Research F, vol. 12, no. 3, pp. 242-255, 2009.

[12] R. G. Zhou, W. J. Horrey, and R. F. Yu, "The effect of conformity tendency on pedestrians' road-crossing intentions in China: an application of the theory of planned behavior," Accident Analysis and Prevention, vol. 41, no. 3, pp. 491-497, 2009.

[13] G. Tiwari, S. Bangdiwala, A. Saraswat, and S. Gaurav, "Survival analysis: pedestrian risk exposure at signalized intersections," Transportation Research F, vol. 10, no. 2, pp. 77-89, 2007.

[14] M. Johnson, J. Charlton, and J. Oxley, "Cyclists and red lighta study of behaviour of commuter cyclists in Melbourne," in Proceedings of the Australasian Road Safety Research, Policing and Education Conference, Adelaide, Australia, November 2008.

[15] M. Johnson, S. Newstead, J. Charlton, and J. Oxley, "Riding through red lights: the rate, characteristics and risk factors of non-compliant urban commuter cyclists," Accident Analysis and Prevention, vol. 43, no. 1, pp. 323-328, 2011.

[16] M. Johnson, J. Charlton, J. Oxley, and S. Newstead, "Why do cyclists infringe at red lights? An investigation of Australian cyclists' reasons for red light infringement," Accident Analysis and Prevention, vol. 50, no. 1, pp. 840-847, 2013.

[17] C. R. Bhat, "Duration modeling," in Handbook of Transport Modelling, D. A. Hensher, D. A. Hensher, and K. J. Button, Eds., pp. 91-111, Elsevier Science, Oxford, UK, 2000.

[18] K. M. N. Habib, "Modeling commuting mode choice jointly with work start time and work duration," Transportation Research A, vol. 46, no. 1, pp. 33-47, 2012.

[19] Y. Chung, "Development of an accident duration prediction model on the Korean freeway systems," Accident Analysis and Prevention, vol. 42, no. 1, pp. 282-289, 2010.

[20] X. B. Yang, Z. Y. Gao, H. W. Guo, and M. Huan, "Survival analysis of car travel time near a bus stop in developing countries," Science in China E, vol. 55, no. 8, pp. 2355-2361, 2012.

[21] X. B. Yang, M. Huan, H. W. Guo, and L. Gao, "Car travel time estimation near a bus stop with non-motorized vehicles," International Journal of Computational Intelligence Systems, vol. 6, no. 4, pp. 1350-1357, 2011.

[22] H. W. Guo, W. H. Wang, W. W. Guo, and F. C. Zhao, "Modeling lane-keeping behavior of bicyclists using survival analysis 
approach," Discrete Dynamics in Nature and Society, vol. 2013, Article ID 197518, 6 pages, 2013.

[23] H. W. Guo, W. H. Wang, W. W. Guo, X. B. Jiang, and H. Bubb, "Reliability analysis of pedestrian safety crossing in urban traffic environment," Safety Science, vol. 50, no. 4, pp. 968-973, 2012.

[24] X. B. Yang, M. Huan, B. F. Si, L. Gao, and H. W. Guo, "Crossing at a red light: behavior of cyclists at urban Intersections," Discrete Dynamics in Nature and Society, vol. 2012, Article ID 490810, 12 pages, 2012.

[25] E. T. Lee and J. W. Wang, Statistical Methods for Survival Data Analysis, John Wiley \& Sons, New York, NY, USA, 2003.

[26] W. H. Wang, H. W. Guo, Z. Y. Gao, and H. Bubb, "Individual differences of pedestrian behaviour in midblock crosswalk and intersection," International Journal of Crashworthiness, vol. 16, no. 1, pp. 1-9, 2011.

[27] L. Yao, L. S. Sun, Z. Y. Zhang, S. W. Wang, and J. Rong, "Research on the behavior characteristics of pedestrian crowd weaving flow in transport terminal," Mathematical Problems in Engineering, vol. 2012, Article ID 264295, 9 pages, 2012. 


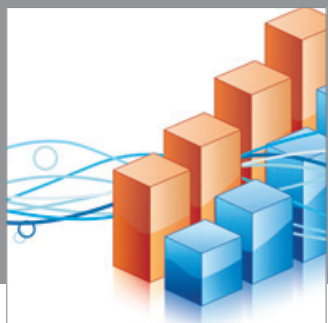

Advances in

Operations Research

mansans

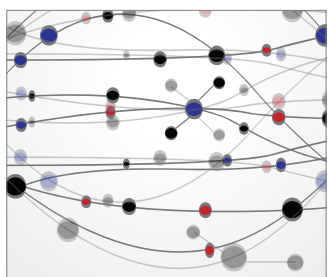

The Scientific World Journal
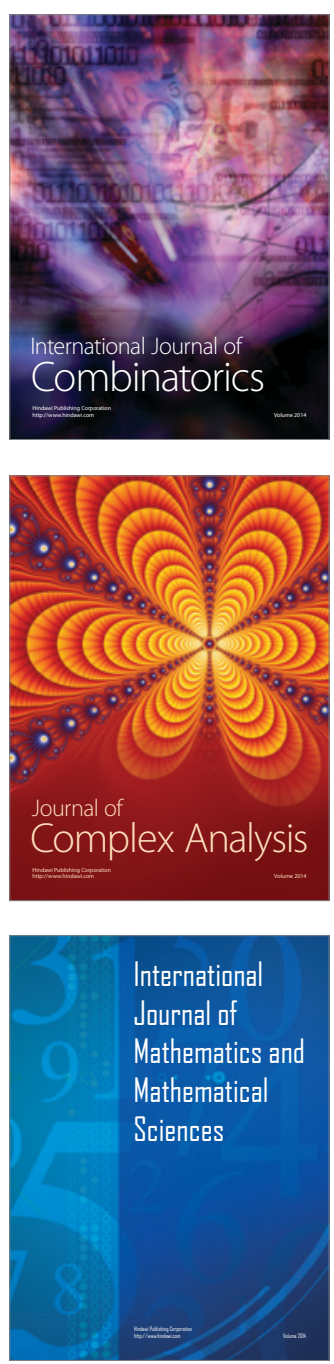
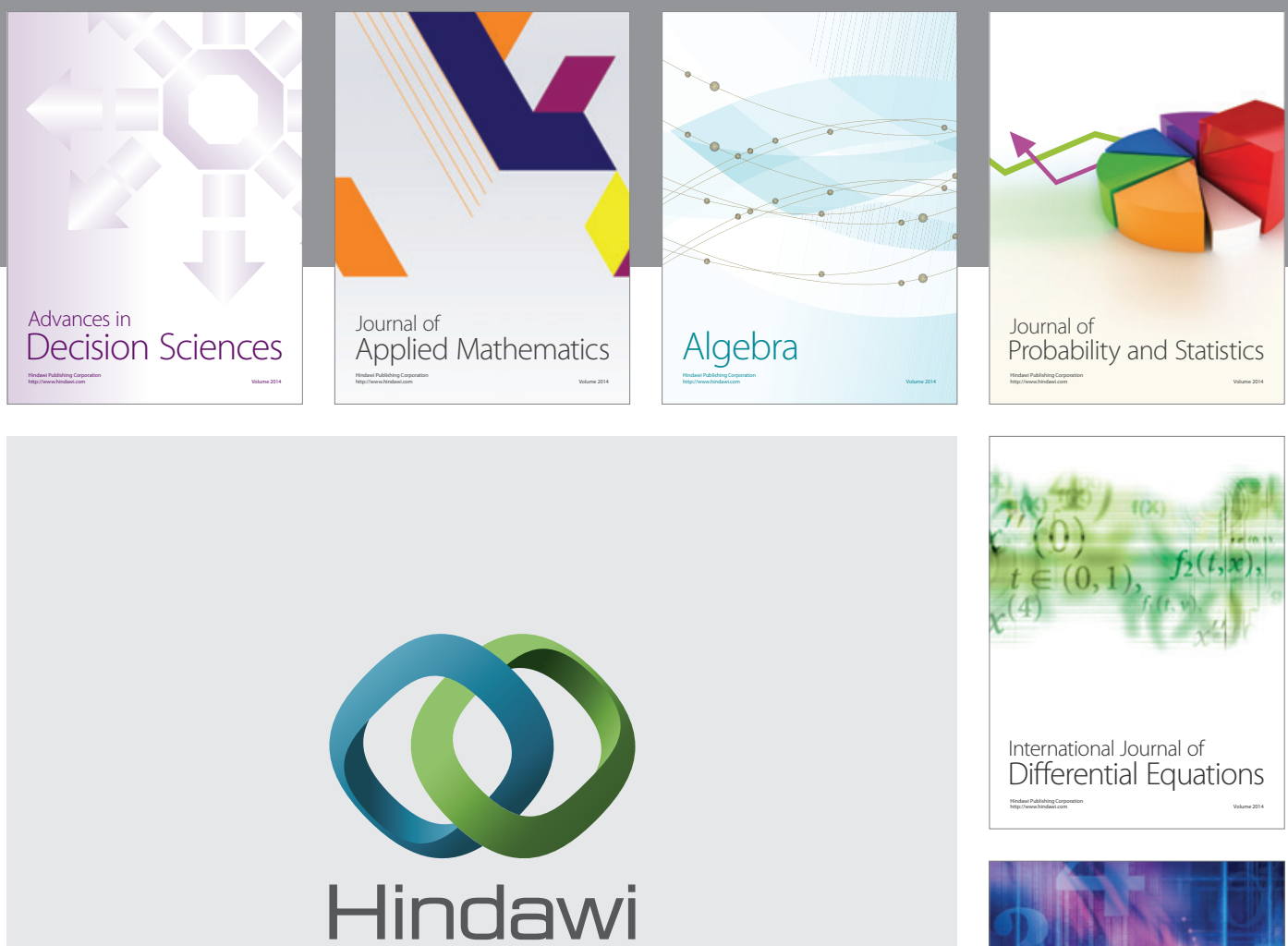

Submit your manuscripts at http://www.hindawi.com
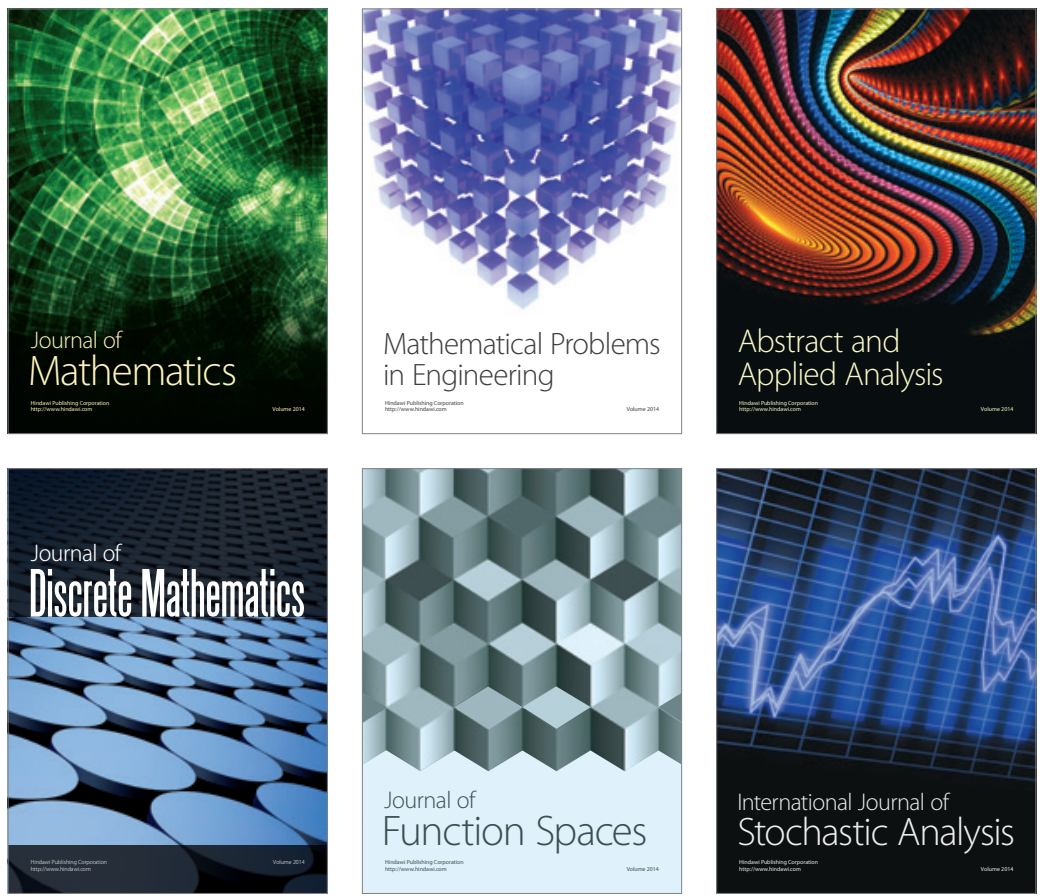

Journal of

Function Spaces

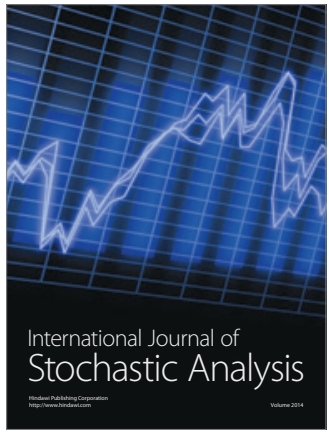

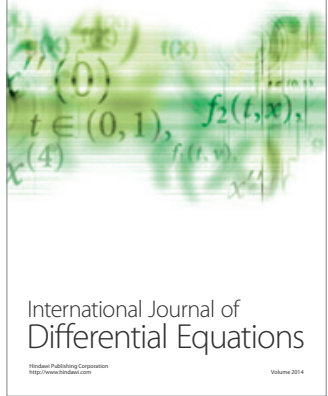
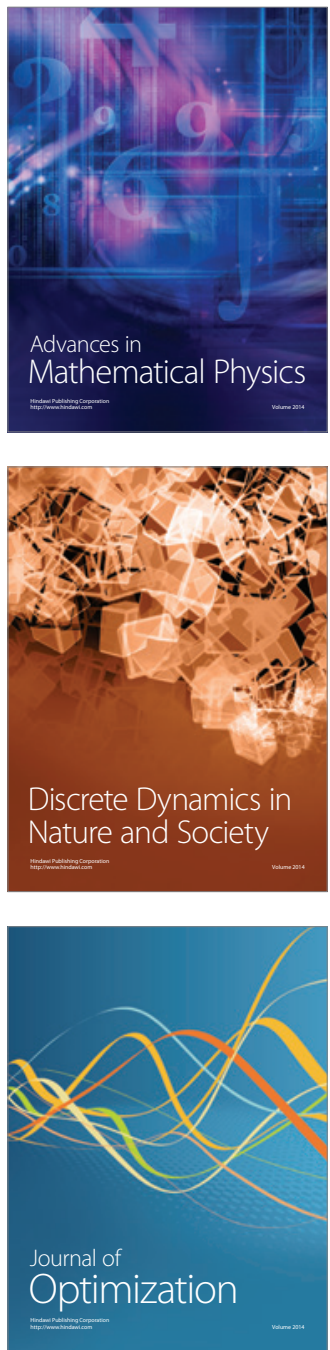